\title{
Functional and diffusion tensor magnetic resonance imaging of the sheep brain
}

\author{
Wonhye Lee ${ }^{1}$, Stephanie D. Lee ${ }^{1}$, Michael Y. Park', Lori Foley², Erin Purcell-Estabrook², Hyungmin Kim ${ }^{1,3}$ \\ and Seung-Schik Yoo ${ }^{1 *}$
}

\begin{abstract}
Background: An ovine model can cast great insight in translational neuroscientific research due to its large brain volume and distinct regional neuroanatomical structures. The present study examined the applicability of brain functional magnetic resonance imaging (fMRI) and diffusion tensor imaging (DTI) to sheep using a clinical MR scanner (3 tesla) with a head coil. The blood-oxygenation-level-dependent (BOLD) fMRI was performed on anesthetized sheep during the block-based presentation of external tactile and visual stimuli using gradient echo-planar-imaging (EPI) sequence.

Results: The individual as well as group-based data processing subsequently showed activation in the eloquent sensorimotor and visual areas. DTI was acquired using 26 differential magnetic gradient directions to derive directional fractional anisotropy (FA) and apparent diffusion coefficient (ADC) values from the brain. White matter tractography was also applied to reveal the macrostructure of the corticospinal tracts and optic radiations.
\end{abstract}

Conclusions: Utilization of fMRI and DTI along with anatomical MRI in the sheep brain could shed light on a broader use of an ovine model in the field of translational neuroscientific research targeting the brain.

Keywords: Sheep, Sensorimotor, Visual, fMRI, DTI, Tractography

\section{Background}

Ovine models for neurological application targeting the brain have been gaining momentum for providing translational information on therapeutic applications to humans. Compared to small animal testing [1], the ovine model of medical research provides important translational information that contributes meaningful insights to human studies. Unlike pigs, which have a flat and thick skull [2], sheep have a relatively round skull that provides comparable structural proximity to the human skull. With large brain volume and distinctive neuro-anatomical structures, the use of the ovine models has been continuing to expand in neuroscience research fields, for example, stroke [3], epilepsy [4], and brain injury models [5]. Emerging therapeutic applications, such as non-invasive focused ultrasound surgeries to ablate soft/tumor tissue [6,7], would also benefit from using the ovine model.

\footnotetext{
* Correspondence: yoo@bwh.harvard.edu

'Department of Radiology, Brigham and Women's Hospital, Harvard Medical School, Boston, MA, USA

Full list of author information is available at the end of the article
}

Anatomical and functional neuroimaging techniques are widely used to characterize functional-structural correlates or any morphological abnormalities in the brain that are implied in various pathological processes. Among various imaging modalities, magnetic resonance imaging (MRI) is advantageous in examining the brain anatomy compared to computerized tomography $(\mathrm{CT})$ which provides limited signal contrasts for soft tissue. MRI, by utilizing a blood-oxygenation-level-dependent (BOLD) endogenous contrast mechanism associated with local brain activations, can also reveal spatiotemporal pattern of regional brain function in response to external stimulation in anesthetized animals (referred as functional MRI; fMRI).

MRI also offers information on the white matter (WM) structures and their spatial orientations. Diffusion tensor MRI (DTI), introduced in the mid 90s [8, 9], uses multiple diffusion-sensitive pulsed-gradient pairs with differing directions that provide characterization of the diffusion of proton-bearing molecules along three orthogonal directions in each voxel. Computed diffusion tensor eigenvectors represent the voxel-specific orthogonal principal axes 
of diffusion, in which each respective eigenvalue reflects the amount of diffusivity in these axes [10]. Apparent diffusion can then be quantitatively analyzed, conveying intra-voxel information about the averaged local (intracellular and extracellular) water diffusion in the brain tissue. Combined with tractography technique [11-13], therefore, DTI offers information on macroscopic orientations in the WM tracts.

While these techniques have well been established and widely used in humans $[14,15]$ and animals (such as rats $[16,17]$, cats $[18,19]$, or dogs $[20,21]$, the application in ovine has rarely been performed. Opdam et al. [22] showed concurrent fMRI and EEG data acquisition in a sheep epilepsy model to localize the epileptic locus. Boltze et al. [3] used MRI and PET along with CT for neuroimaging of a sheep stroke model. A recent work by Hoffmann et al. [23] presented a combination of anatomical MRI and CT data along with cranial anatomy, focusing on the ovine cerebrovascular system. While these previous studies showed the potential utilization of neuroradiological imaging in ovine, only a limited amount of information on the healthy brain was available. Therefore, we were motivated to examine the feasibility of applying a routine human neuro MRI protocol to image the normal sheep brain. Anatomical MRI as well as functional MRI (fMRI) was conducted during photic stimulation (presented through closed eyelid) and tactile stimulation (on the unilateral hind leg). The photic and tactile stimulations are some of the earliest stimulation modalities used in the context of fMRI, and are widely adopted in studies involving humans [24-27] and small animals $[28,29]$. Because these stimulation modalities can activate the primary sensory circuities (such as primary sensory and visual areas) even during the anesthesia $[28,30]$, they are compatible with animal studies. Besides fMRI, voxel-wise apparent diffusion coefficient (ADC), along with fractional anisotropy (FA; as a measure of the directionality of diffusion) values were obtained using DTI, and the spatial orientations of the corticospinal tracts and optic radiations were visualized using tractography technique.

\section{Results}

\section{Example of individual T1, T2, fMRI, and 3D SPGR high resolution $\mathrm{MRI}$ rendering}

All animals successfully underwent the MRI session. Exemplar T1- and T2-weighted axial images of the sheep brain are shown in Fig. 1a. In the T1 images, small vascular flow artifacts were visible along the phase-encoding direction (marked with arrows) due to the vascular anatomy on ovine facial area (angularis oculi vein $[31,32])$. The activated functional areas, from the sensorimotor stimulation (Fig. 1b) and visual stimulation (Fig. 1c) of one sheep, were visualized (uncorrected $P<0.001$, with a cluster extent threshold of 27 continuous voxels) and overlaid on axial, coronal, and sagittal slices, along with a 3D-rendered head view.

\section{fMRI group processing results and the time-course of BOLD signals}

fMRI group processing results $(n=7)$ that identified the activated sensorimotor $(P<0.05$, no voxel-based corrections) and visual areas (uncorrected $P<0.01$, with a cluster extent threshold of 27 continuous voxels) are shown in Fig. 2a. The location and the size of the activated regions from the group analysis according to the different statistical properties (with and without the extent threshold correction) were tabulated (Table 1). Due to the small SM1 area for the hind leg among sheep [33], no additional extent correction was performed when thresholded at $P<0.05$. The activation map was overlaid and visualized on axial, coronal, and sagittal slices of the high resolution 3D SPGR image of an individual sheep. The time-courses of BOLD signals (averaged across seven sheep) from the sensorimotor and visual ROIs are shown in Fig. 2b. The variabilities in spatial distribution of activation among sheep $(n=7)$, as measured from the coordinates showing the local maximum in the voxelwise $t$-value, were quantified by taking the standard deviation along the left-right, rostral-caudal, and dorsalventral directions $(5.40,6.25$, and $5.70 \mathrm{~mm}$ for the sensorimotor area, and 5.32, 3.93, and $3.40 \mathrm{~mm}$ for the visual area). The time delay of the observed BOLD signal was approximated at 3-4 s from the onset of the stimulation with the magnitude of $\sim 3 \%$ (in case of the sensorimotor stimulation) and $\sim 2 \%$ (in case of the visual stimulation) of the baseline signal level.

\section{FA and ADC values and tractography of corticospinal tracts in sheep}

The calculated color-coded FA maps, showing the fiber orientations (Fig. 3a, upper low), and ADC maps (Fig. 3a, lower low) were shown in axial view, along with the selected ROIs used to measure the values. For the colorcoding scheme, red, green, and blue colors were used for left-right, dorsal-ventral, and rostral-caudal directions, respectively. The mean FA and ADC values measured from the selected ROIs were tabulated in Table 2.

In the corpus callosum (CC) genu (Fig. 3a, ROI \#1), the FA value was $0.39 \pm 0.05$ (mean $\pm \mathrm{SD}$ ) and the corresponding ADC value was $1.14 \pm 0.16 \times 10^{-3} \mathrm{~mm}^{2} / \mathrm{s}$ (mean $\pm \mathrm{SD}$ ). The FA value measured from the CC splenium (Fig. 3a, ROI \#3) was $0.37 \pm 0.16$ and the ADC value was $0.91 \pm$ $0.07 \times 10^{-3} \mathrm{~mm}^{2} / \mathrm{s}$. The greatest FA values were observed from the internal capsules (Fig. 3a, ROI \#2), which were $0.63 \pm 0.13$ for the left and $0.65 \pm 0.16$ for the right. The corresponding mean ADC values were $0.84 \pm 0.21 \times 10^{-3}$ and $0.75 \pm 0.12 \times 10^{-3} \mathrm{~mm}^{2} / \mathrm{s}$. From the ROIs for the 

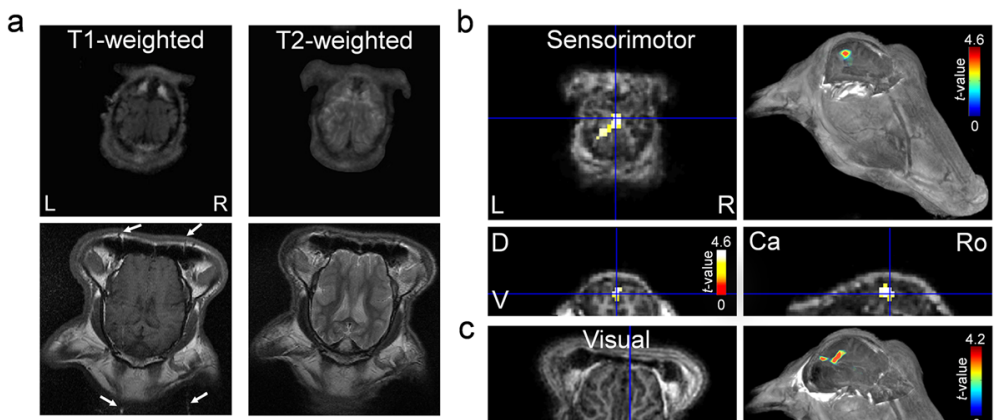

C
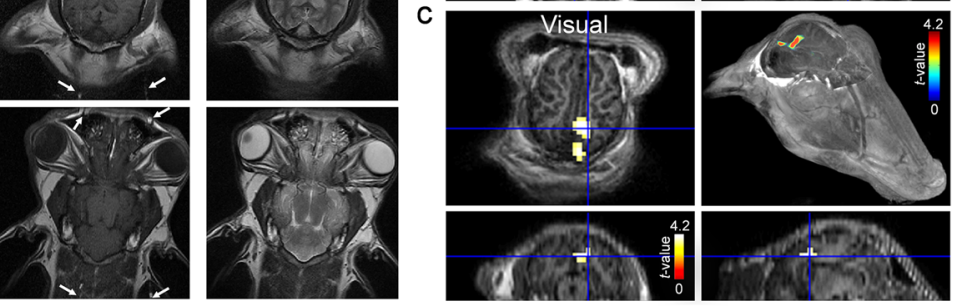

Fig. 1 Example of MRI data from an individual sheep. a T1-weighted (left column) and T2-weighted (right column) axial images from dorsal (top) to ventral (bottom). In the T1 images, arrows indicate flow-related artifacts along the phase-encoding direction. Individual fMRI activation maps from (b) the sensorimotor and (c) visual stimulations were overlaid on axial, coronal, and sagittal views as well as on a 3D MRI head rendering. Crosshairs (in blue) indicate the location of the local maxima on the fMRI activation map from the square frame (in blue) showed on an axial plane of the 3D rendering. L: left, R: right, D: dorsal, V: ventral, Ca: caudal, and Ro: rostral

corona radiata (Fig. 3a, ROI \#4), the mean FA values were $0.48 \pm 0.13$ for the left and $0.46 \pm 0.18$ for the right, while the corresponding mean ADC values were $0.80 \pm 0.07 \times$ $10^{-3}$ and $0.89 \pm 0.14 \times 10^{-3} \mathrm{~mm}^{2} / \mathrm{s}$, respectively. In the analysis of interhemispheric differences, no significant differences were found in both FA and ADC values in the internal capsule (paired $t$-test, two-tailed, $P=0.67$ for FA, $P=0.20$ for ADC, $n=6)$ or from the corona radiata (paired $t$-test, two-tailed, $P=0.60$ for FA, $P=0.21$ for ADC, $n=6$ ). DTI tractography performed on the DTI data visualized the corticospinal tracts and optic radiations bilaterally (Fig. 3b), with the seeding areas of the internal capsule and thalamic lateral geniculate areas for the tracking, respectively.

\section{Discussion}

The present study demonstrated the applicability of MR imaging for non-invasive neuro-functional assessment in a healthy sheep model. fMRI was employed to examine the sensorimotor and visual cortex activated by external sensory stimuli in healthy sheep. The identified functional areas were in a good agreement with the areas probed by using cortical stimulation of the motor area [33] and visual photic stimulation [34]. Also, the measurements taken from DTI (FA and ADC values) as well as the tractography showed the feasibility of examining the WM macrostructures.

The time-course of BOLD signals obtained from the sheep (Fig. 2b) showed contrast magnitude on the order
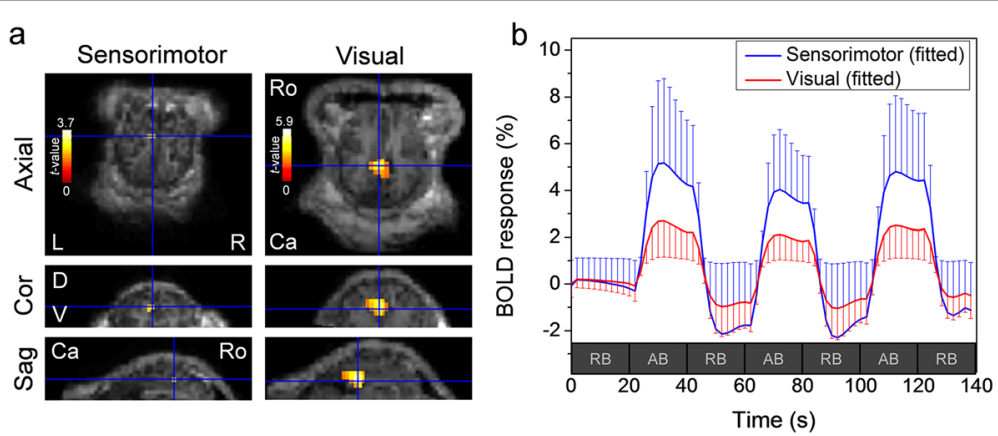

Fig. 2 Group-processed fMRI activation maps and the time-course of BOLD signals. a Group-level fMRI activation maps from seven sheep via random-effect analysis of the sensorimotor area $(P<0.05$, no voxel-based corrections) and visual area (uncorrected $P<0.01$, with cluster extent threshold of 27 contiguous voxels) were overlaid on axial, coronal and sagittal anatomical MRI. Left column: via sensorimotor stimulation, Right column: via visual stimulation. L: left, R: right, D: dorsal, V: ventral, Ca: caudal, and Ro: rostral. b The time-course of BOLD responses averaged across the group of sheep ( $n=7$, error bars are standard deviation of the signal) from the local maxima of sensorimotor-(blue) and visual-(red) related region-of-interests (ROIs) on fMRI activation maps. RB: resting block (20 s-long), AB: activation block (20 s-long) 
Table 1 The location and the size of the activated regions from group analysis $(n=7)$ with the different statistical properties

\begin{tabular}{|c|c|c|c|c|}
\hline \multirow[b]{2}{*}{ P-value } & \multicolumn{2}{|c|}{ Sensorimotor stimulation } & \multicolumn{2}{|c|}{ Visual stimulation } \\
\hline & $\begin{array}{l}\text { Activated } \\
\text { region }\end{array}$ & Voxel size & $\begin{array}{l}\text { Activated } \\
\text { region }\end{array}$ & $\begin{array}{l}\text { Voxel } \\
\text { size }\end{array}$ \\
\hline $\begin{array}{l}0.05 \\
\text { (Uncorrected) }\end{array}$ & SM1 & 3 & V1 & 212 \\
\hline 0.05 (Corrected) & - & 0 & V1 & 212 \\
\hline 0.01 (Corrected) & - & 0 & V1 & 36 \\
\hline
\end{tabular}

Corrected: application of a cluster extent threshold of 27 continuous voxels, SM1: primary sensorimotor area, V1: primary visual area

of $2-3 \%$ (from the resting signal level) and an onset response delay of 3-4 s, exhibiting similarities to the signals from humans [35-37]. Since the current fMRI protocol (with TR of $2 \mathrm{~s}$ ) lacks the temporal precision to produce an accurate representation of the signal response delay, a study employing the use of a shorter TR (on the order of less than $1 \mathrm{~s}$ ) combined with the event-related designs [38, 39] would reveal more

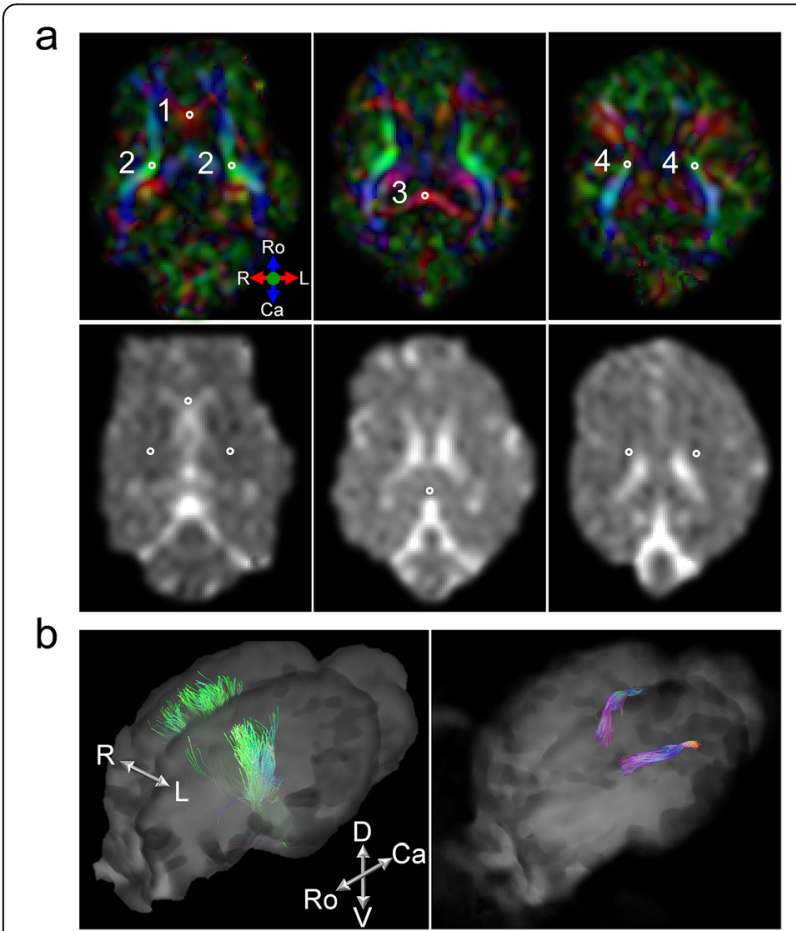

Fig. 3 The processed DTI data to visualize white matter tracts in the brain. a The placement of the regions of interests (ROIs) on the colorcoded fractional anisotropy (FA) map and the corresponding apparent diffusion coefficient (ADC) map. \#1: genu of corpus callosum, \#2: internal capsule, \#3: splenium of corpus callosum, \#4: corona radiate. The regions outside of the brain parenchyma were masked out from the display. For the FA color-coding scheme, red, green, and blue colors were used for left-right, dorsal-ventral, and rostral-caudal directions, respectively. b Bilaterally visualized DTI tractography of the corticospinal tracts and optic radiations in sheep (software-generated pseudo-coloring was used) comprehensive information on the BOLD hemodynamic properties from the sheep. It is also important to note that the BOLD response is affected by the anesthetic depth or by type of anesthetic agents due to potential alterations of the hemodynamic response to the neuronal activation [40-42]. Further study is warranted to examine the effects of different anesthetic agents, other than Telazol, such as propofol infusion [43-45], on the BOLD signal responses and the central nervous system in ovine species.

In the interpretation of the DTI data, both FA and ADC values from the ROIs in sheep (the CC genu and splenium, and the internal capsule, shown in Fig. 3a) were qualitatively comparable to the data from healthy human adults reported by Brander et al. [46] (Table 2). On the other hand, the FA values measured from sheep's corona radiata (left: 0.48 and right: 0.46 ) were comparable to those obtained from humans (left: 0.52 and right: 0.48) [46]. In the interhemispheric differences in sheep, there were no significant differences in both FA and ADC values from the internal capsule and the corona radiata, while significant left/right asymmetries from those regions have been reported in humans [46].

Additionally, the FA values from the CC genu and splenium, which are typically shown to be greater than the values from the internal capsule and the corona radiata among humans [46], were smaller than the values from other ROIs (Fig. 3a). We also noted greater ADC values from these areas compared to the ones from humans. We conjecture that these discrepancies may be attributed to the partial volume effects caused by the relatively thick imaging slice $(3 \mathrm{~mm})$ and large voxel volume with respect to the ovine neuroanatomy.

DTI tractography successfully visualized the corticospinal tracts and optic radiations in sheep (Fig. 3b), but was inadequate to visualize other WM tracts such as the frontoparietal fasciculus, which are readily characterized through the human version of the imaging protocol. The insufficient image-resolution (slice thickness on the order of $3 \mathrm{~mm}$ ) and signal-to-noise ratio (SNR) were considered as the causes of the limited ability to track major WM tracts. The use of a head coil that is designed for a human head might also have resulted in the lack of SNR of the acquired neuroanatomical data (due to smaller voxel size compared to the human counterparts due to use of smaller field-ofview). The use of higher spatial resolution (i.e., smaller voxel size, ideally isotropic in volume) combined with a dedicated head coil having smaller coil elements to boost SNR (to compensate for the decrease in the MR signal associated with the higher spatial resolution) would be conducive to identifying small WM bundles in sheep. The use of a sheep-dedicated head coil would not only improve the SNR but would also provide more 
Table 2 Regional mean values of fractional anisotropy (FA) and apparent diffusion coefficient (ADC)

\begin{tabular}{|c|c|c|c|c|c|c|c|}
\hline \multicolumn{4}{|c|}{ (a) Sheep 3 T $(n=6)$} & \multicolumn{4}{|c|}{ (b) Human $3 \mathrm{~T}(n=30)$} \\
\hline \multirow[b]{3}{*}{ Region } & & FA & $A D C$ & \multirow[b]{3}{*}{ Region } & & $\mathrm{FA}$ & $A D C$ \\
\hline & & \multicolumn{2}{|c|}{$\times 10^{-3} \mathrm{~mm}^{2} / \mathrm{s}$} & & & \multicolumn{2}{|c|}{$\times 10^{-3} \mathrm{~mm}^{2} / \mathrm{s}$} \\
\hline & & Mean \pm SD & Mean \pm SD & & & Mean \pm SD & Mean \pm SD \\
\hline$\overline{C C}$ genu & & $0.39 \pm 0.05$ & $1.14 \pm 0.16$ & CC genu & & $0.84 \pm 0.03$ & $0.76 \pm 0.04$ \\
\hline CC splenium & & $0.37 \pm 0.16$ & $0.91 \pm 0.07$ & CC splenium & & $0.86 \pm 0.05$ & $0.71 \pm 0.03$ \\
\hline \multirow[t]{2}{*}{ Internal capsule } & Left & $0.63 \pm 0.13$ & $0.84 \pm 0.21$ & Internal capsule & Left & $0.75 \pm 0.04$ & $0.68 \pm 0.03$ \\
\hline & Right & $0.65 \pm 0.16$ & $0.75 \pm 0.12$ & & Right & $0.75 \pm 0.05$ & $0.70 \pm 0.03$ \\
\hline \multirow[t]{2}{*}{ Corona radiata } & Left & $0.48 \pm 0.13$ & $0.80 \pm 0.07$ & Corona radiata & Left & $0.52 \pm 0.05$ & $0.65 \pm 0.04$ \\
\hline & Right & $0.46 \pm 0.18$ & $0.89 \pm 0.14$ & & Right & $0.48 \pm 0.04$ & $0.66 \pm 0.03$ \\
\hline
\end{tabular}

FA and ADC from (a) sheep DTI data $(n=6)$ and (b) human DTI data (adapted from Brander et al. [46]), acquired with 3 T MRI. CC: corpus callosum

homogenous signal sensitivity profile across the brain volume.

In the review of overall experimental procedures, we experienced a few technical difficulties, especially from the head movement of the anesthetized sheep during the MRI session(s), which considerably reduced the quality of the image in couple of animals. Although restrainers placed over the sheep head (i.e., use of cushion between the sheep head and a head coil) were helpful in reducing the artifacts, bloating of the abdomen could have amplified breathing-related head motion beyond the restraining capacity. Since ruminants are especially susceptible to bloating, the use of intubation would be beneficial to prevent the bloating of stomach during imaging procedures.

We also noted the artifacts in T1 images from the sheep (Fig. 1a, arrows), which were likely attributable to the vascular feature (angularis oculi veins [31, 32]) that prominently exists in the ovine facial area but not in humans. The artifacts call for necessary countermeasures, such as introduction of additional flow-suppression [47] or cardiac gating [48], when using human-compatible imaging protocol on ovine species.

\section{Conclusions}

Ovine model can provide a unique opportunity for testing new neurotherapeutic modalities that can potentially be translated to clinical applications in humans, for example, to focused ultrasound (FUS) brain stimulation technology which has shown evidences in neuromodulatory potentials in small animal models [1]. Applicability of MRI, fMRI, and DTI in the sheep brain, as demonstrated in the present study, could further enhance the range of utilization of an ovine model in the field of translational neuroscientific research targeting the brain.

\section{Methods}

\section{Overview}

The study was conducted under the approval of the Harvard Medical Area Standing Committee on Animals.
Sheep (Dorset, all female, 20-40 kg, $n=8$ ) were anesthetized with Telazol (Tiletamine; N-methyl-D-aspartate; NMDA receptor antagonist + zolazepam, initial dose $2-4 \mathrm{mg} / \mathrm{kg}$ i.v. + additional doses as needed) for the MRI procedures. The use of inhalant anesthetics, such as isoflurane, was not chosen due to its effects on altering the cerebral blood hemodynamic responses, such as vasodilation [49, 50], which confound the BOLD signal. The adequacy of anesthesia was assessed by the absence of the reflex withdrawal of the hind limb in response to pinching, and by monitoring the heart rate and peripheral capillary oxygen saturation level $\left(\mathrm{SpO}_{2}\right)$ during the procedure. The anesthetic agent was administered only to those animals with normal vital signs. Cushions were placed between the sheep head and a head coil to discourage movement and subsequent motion-related MR artifacts.

\section{Data acquisition}

MRI was performed to obtain the anatomical information of the sheep brain. A 3 tesla clinical MRI scanner (GE VH, Waukesha, WI) with an 8-channel head coil was used. Anatomical T1- and T2-weighted images (spin echo; SE for T1 and fast spin echo; FSE for T2, field-ofview; FOV $=18 \times 18 \mathrm{~cm}^{2}$, slice thickness $=3 \mathrm{~mm}$, image matrix $=512 \times 512$, voxel size $=0.35 \times 0.35 \times 3 \mathrm{~mm}^{3}$, TR/ $\mathrm{TE}=567 / 9 \mathrm{~ms}$ for $\mathrm{T} 1$ and $\mathrm{TR} / \mathrm{TE}=2783 / 100 \mathrm{~ms}$ for $\mathrm{T} 2$, flip angle $=65^{\circ}$ ) were acquired as sections of the ventral-dorsal orientation with respect to the animal's head covering the entire brain area. To provide high resolution anatomical information, we also acquired volumetric T1weighted images covering most of the head including the brain (inversion recovery; IR - 3D spoiled gradient recalled sequence; SPGR, orientation in $3 \mathrm{D}$ blocked sagittal, FOV $=22 \times 22 \mathrm{~cm}^{2}$, slice thickness $=1 \mathrm{~mm}$, image matrix $=256 \times 256$, voxel size $=0.86 \times 0.86 \times 1 \mathrm{~mm}^{3}$, TR/ $\mathrm{TE}=7 / 3 \mathrm{~ms}$, flip angle $=11^{\circ}$ ).

Functional MRI (fMRI) was performed to map the sensorimotor and visual areas of the sheep brain, using 
gradient-echo echo-planar-imaging (EPI) sequence (TR/ $\mathrm{TE}=2000 / 40 \mathrm{~ms} ;$ flip angle $=90^{\circ} ; \mathrm{FOV}=18 \times 18 \mathrm{~cm}^{2}$; image matrix $=64 \times 64$; slice thickness $=3 \mathrm{~mm}$; slices $=$ 20 , no gap; voxel size $=2.81 \times 2.81 \times 3.00 \mathrm{~mm}^{3}$ ) to image the brain as sections of the ventral-dorsal orientation with respect to the animal's head for obtaining the T2*weighted blood-oxygenation-level-dependent (BOLD) signal. Although TE of 30-35 ms is widely used in fMRI at $3 \mathrm{~T}$ environment, a slightly longer TE was used in this study to maximize the sensitivity toward the BOLD contrast from the sheep [51]. The scanning orientation was set using the same scan prescription location as the anatomical T1- and T2-weighted brain images. The activations of the sensorimotor and visual areas were evoked using a passive tactile stimulation (i.e., gentle $2 \mathrm{~Hz}$ squeeze of the right hind leg muscle) and a photic stimulation (i.e., $2 \mathrm{~Hz}$ white strobe lights to both eyes with eyelids closed), respectively. Three blocks of the stimulation period $(20 \mathrm{~s}$, synchronized with the scanner operation) were interleaved by four resting periods of equal duration $(20 \mathrm{~s})$. A dummy scan of $20 \mathrm{~s}$ was included in the beginning of the imaging session to allow for T1 signal equilibration, but was not used in the data processing.

A diffusion tensor imaging (DTI) sequence (spin echo; SE, echo-planar-imaging; EPI, oblique axial orientation, FOV $=18 \times 18 \mathrm{~cm}^{2}$, slice thickness $=3 \mathrm{~mm}$, image matrix $=256 \times 256$, voxel size $=0.7 \times 0.7 \times 3.0 \mathrm{~mm}^{3}$, TR/ $\mathrm{TE}=6000 / 90 \mathrm{~ms}$, flip angle $=90^{\circ}$, acceleration factor $=2$; 26 gradient directions, $\mathrm{b}$-factor $=1000$ ) was used to examine FA and ADC values of the sheep brain. 26 nonoverlapping gradient directions were used to probe the spatial orientation of voxel-wise water diffusivity. All imaging parameters were similar to the ones used in an adult human, however, a smaller FOV (i.e., $18 \times 18 \mathrm{~cm}^{2}$ ) was used compared to the one used in human studies (i.e., $22 \times 22 \mathrm{~cm}^{2}$ ).

\section{fMRI data processing}

The fMRI time series from seven sheep were used for the data processing. Data from one sheep were excluded due to severe motion-related artifacts ( $>5 \mathrm{~mm}$ in translational motion) during the scan. The time series were processed by the SPM8 software package (Wellcome Department of Imaging Neuroscience, University College London, London, UK; www.fil.ion.ucl.ac.uk/spm). The datasets obtained from the group of sheep underwent slice time correction, and were neuroanatomically coaligned for motion correction. Then, the datasets were spatially smoothed with the Gaussian kernel having a full-width at half maximum (FWHM) of $[8 \times 8 \times 8]$ in $\mathrm{mm}$. After the motion-correction and smoothing, the task-related neuronal activity was estimated by a general linear model (GLM) for each individual sheep. The degree of voxel-wise statistic parametric map in $t$-value, with respect to the task-specific canonical hemodynamic response function (HRF), was obtained. To derive the group-averaged trend, random effect analysis (RFX) [52] was used to perform a statistical test at the group level. To reject false positives based on cluster extent thresholds [53] in the statistical parametric map, the cluster size was calculated using the REST AlphaSim toolbox [54] (www.restfmri.net; toolkit v1.8; 1000 Monte Carlo simulations were used). The time-course of BOLD signals from the region-of-interests (ROIs) for the sensorimotor and visual areas were averaged across the animals $(n=7)$.

\section{DTI data processing}

Data from two animals, one due to motion effects and the other due to technical issues, were excluded. The acquired DTI sequence $(n=6)$ was processed by the DTI Studio software (Department of Radiology, Johns Hopkins University, Baltimore, MD), whereby diffusion tensor calculation was performed with diffusion-weighted image parameters (slice orientation: axial, slice sequencing: inferior-superior, b-value $=1000 \mathrm{~s} / \mathrm{mm}^{2}$ ). Fractional anisotropy (FA), apparent diffusion coefficient (ADC), trace, tensor, and color-coded directional FA maps were calculated as provided in the software. FA and ADC are one of the most frequently used quantitative parameters in humans. Circular region-of-interests (ROIs), having a size of 9 pixels, were delineated based on the directional FA maps. The ROIs were placed on the following anatomical locations (also shown in Fig. 3a): on the genu and splenium of the corpus callosum (CC), on the left and right internal capsule, and on the left and right corona radiate, and the FA and ADC values from these ROIs were averaged (Table 2). The ADC value was calculated as the trace of the tensor $(\operatorname{Tr})$ divided by three $(\mathrm{ADC}=\operatorname{Tr} / 3)$ [55]. To demonstrate the feasibility of DTI tractography, white matter tracking routine, as implemented by the MRtrix [56, 57], was employed using the software options (start tracking criteria FA $>0.3$; stop tracking criteria FA < 0.3 and fiber turning angular tolerance of $20^{\circ}$ ). The seeding areas were defined in the internal capsule and thalamic lateral geniculate areas for the tracking and visualization of the corticospinal tracts and optic radiations, respectively.

\section{Abbreviations}

ADC: Apparent diffusion coefficient; BOLD: Blood-oxygenation-level-dependent; CC: Corpus callosum; CT: Computerized tomography; DTI: Diffusion tensor MRI; EPI: Echo-planar-imaging; FA: Fractional anisotropy; fMRI: Functional MRI; FWHM: Full-width at half maximum; GLM: General linear model; HRF: Hemodynamic response function; MRI: Magnetic resonance imaging; RFX: Random effect analysis; ROI: Region-of-interest; SNR: Signal-to-noise ratio; TE: Echo time; TR: Repetition time; WM: White matter.

\section{Competing interests}

The authors declare no competing interests. 


\section{Authors' contributions}

SSY conceived of the study; WL, SDL, HK, and SSY designed the study; WL, SDL, MYP, LF, EP, HK, and SSY performed the experiments; WL, HK, and SSY analyzed the data. All authors read, contributed to and approved the final manuscript.

\section{Acknowledgements}

Authors thank Drs. Yongzhi Zhang and Mimi Lam for the helpful technical advice in sheep procedures. Initial help and supports by Mr. Jeffrey Pettit and Ms. Rita G. Laurence are also acknowledged. All the imaging was conducted with the help of the fMRI service team in Brigham and Women's Hospital, Harvard Medical School. Authors also thank Dr. Scott Hoge for the helpful discussion regarding the MRI artifacts. This study was supported by NIH (R21 NSO74124 to S.-S. Yoo), the Focused Ultrasound Surgery Foundation (to S.-S. Yoo), and the Korea Institute of Science and Technology Institutional Program (2E23031 to S.-S. Yoo).

\section{Author details}

'Department of Radiology, Brigham and Women's Hospital, Harvard Medical School, Boston, MA, USA. Invasive Cardiovascular Experimental Laboratory, Brigham and Women's Hospital, Harvard Medical School, Boston, MA, USA.

${ }^{3}$ Center for Bionics, Korea Institute of Science and Technology, Seoul, Korea.

\section{Received: 6 November 2014 Accepted: 12 October 2015}

\section{Published online: 14 October 2015}

\section{References}

1. Yoo S-S, Bystritsky A, Lee J-H, Zhang Y, Fischer K, Min B-K, et al. Focused ultrasound modulates region-specific brain activity. Neurolmage. 2011;56(3):1267-75.

2. Cohen ZR, Zaubermann J, Harnof S, Mardor Y, Nass D, Zadicario E, et al. Magnetic resonance imaging-guided focused ultrasound for thermal ablation in the brain: a feasibility study in a swine model. Neurosurgery. 2007;60(4):593-600. discussion 600.

3. Boltze J, Förschler A, Nitzsche B, Waldmin D, Hoffmann A, Boltze CM, et al. Permanent middle cerebral artery occlusion in sheep: a novel large animal model of focal cerebral ischemia. J Cereb Blood Flow Metab. 2008;28(12):1951-64.

4. Stypulkowski PH, Stanslaski SR, Jensen RM, Denison TJ, Giftakis JE. Brain stimulation for epilepsy - local and remote modulation of network excitability. Brain Stimul. 2014;7(3):350-8.

5. Van den Heuvel C, Blumbergs PC, Finnie JW, Manavis J, Jones NR, Reilly PL, et al. Upregulation of amyloid precursor protein messenger RNA in response to traumatic brain injury: an ovine head impact model. Exp Neurol. 1999;159(2):441-50.

6. Elias WJ, Huss D, Voss T, Loomba J, Khaled M, Zadicario E, et al. A pilot study of focused ultrasound thalamotomy for essential tremor. N Engl J Med. 2013;369(7):640-8.

7. Martin E, Jeanmonod D, Morel A, Zadicario E, Werner B. High-intensity focused ultrasound for noninvasive functional neurosurgery. Ann Neurol. 2009;66(6):858-61.

8. Basser PJ, Mattiello J, LeBihan D. MR diffusion tensor spectroscopy and imaging. Biophys J. 1994;66(1):259-67.

9. Pierpaoli C, Jezzard P, Basser PJ, Barnett A, Di Chiro G. Diffusion tensor MR imaging of the human brain. Radiology. 1996;201(3):637-48.

10. Jellison BJ, Field AS, Medow J, Lazar M, Salamat MS, Alexander AL. Diffusion tensor imaging of cerebral white matter: a pictorial review of physics, fiber tract anatomy, and tumor imaging patterns. AJNR Am J Neuroradiol. 2004:25(3):356-69.

11. Basser PJ, Pajevic S, Pierpaoli C, Duda J, Aldroubi A. In vivo fiber tractography using DT-MRI data. Magn Reson Med. 2000:44(4):625-32.

12. Behrens TE, Berg HJ, Jbabdi S, Rushworth MF, Woolrich MW. Probabilistic diffusion tractography with multiple fibre orientations: What can we gain? Neurolmage. 2007;34(1):144-55.

13. Jiang H, van Zijl PCM, Kim J, Pearlson GD, Mori S. DtiStudio: resource program for diffusion tensor computation and fiber bundle tracking. Comput Methods Programs Biomed. 2006;81(2):106-16.

14. Assaf $Y$, Pasternak $O$. Diffusion tensor imaging (DTI)-based white matter mapping in brain research: a review. J Mol Neurosci. 2007;34(1):51-61.

15. Heeger DJ, Ress D. What does fMRI tell us about neuronal activity? Nat Rev Neurosci. 2002;3(2):142-51.
16. Rumple A, McMurray M, Johns J, Lauder J, Makam P, Radcliffe M, et al. 3dimensional diffusion tensor imaging (DTI) atlas of the rat brain. PLoS ONE. 2013;8(7):e67334.

17. Weber R, Ramos-Cabrer P, Wiedermann D, van Camp N, Hoehn M. A fully noninvasive and robust experimental protocol for longitudinal fMRI studies in the rat. Neurolmage. 2006;29(4):1303-10.

18. Ronen I, Kim K-H, Garwood M, Ugurbil K, Kim D-S. Conventional DTI vs. slow and fast diffusion tensors in cat visual cortex. Magn Reson Med. 2003:49(5):785-90.

19. Jezzard P, Rauschecker JP, Malonek D. An in vivo model for functional MR in cat visual cortex. Magn Reson Med. 1997;38(5):699-705.

20. Lee S-H, Jahng G-H, Choe I-H, Choi C-B, Kim D-H, Kim H-Y. Neural pathway interference by retained acupuncture: a functional MRI study of a dog model of Parkinson's disease. CNS Neurosci Ther. 2013;19(8):585-95.

21. Jacamot O, Van Thielen B, Fierens Y, Hammond M, Willekens I, Van Schuerbeek $P$, et al. Diffusion tensor imaging of white matter tracts in the dog brain. Anat Rec. 2013;296(2):340-9.

22. Opdam HI, Federico P, Jackson GD, Buchanan J, Abbott DF, Fabinyi GC, et al. A sheep model for the study of focal epilepsy with concurrent intracranial EEG and functional MRI. Epilepsia. 2002;43(8):779-87.

23. Hoffmann A, Stoffel MH, Nitzsche B, Lobsien D, Seeger J, Schneider H, et al. The ovine cerebral venous system: comparative anatomy, visualization, and implications for translational research. PLoS ONE. 2014:9(4):e92990.

24. Belliveau JW, Kwong KK, Kennedy DN, Baker JR, Stern CE, Benson R, et al. Magnetic resonance imaging mapping of brain function. Human visual cortex. Invest Radiol. 1992;27 Suppl 2:S59-65.

25. Bandettini PA, Wong EC, Hinks RS, Tikofsky RS, Hyde JS. Time course EPI of human brain function during task activation. Magn Reson Med. 1992;25(2):390-7.

26. Blamire AM, Ogawa S, Ugurbil K, Rothman D, McCarthy G, Ellermann JM, et al. Dynamic mapping of the human visual cortex by high-speed magnetic resonance imaging. Proc Natl Acad Sci U S A. 1992:89(22):11069-73.

27. Yetkin FZ, Mueller WM, Hammeke TA, Morris GL, Haughton VM. Functional magnetic resonance imaging mapping of the sensorimotor cortex with tactile stimulation. Neurosurgery. 1995;36(5):921-5.

28. Lowe AS, Beech JS, Williams SCR. Small animal, whole brain fMRI: innocuous and nociceptive forepaw stimulation. Neurolmage. 2007;35(2):719-28.

29. Fa Z, Zhang P, Huang F, Li P, Zhang R, Xu R, et al. Activity-induced manganese-dependent functional MRI of the rat visual cortex following intranasal manganese chloride administration. Neurosci Lett. 2010;481(2):110-4

30. You Y, Klistorner A, Thie J, Graham SL. Improving reproducibility of VEP recording in rats: electrodes, stimulus source and peak analysis. Doc Ophthalmol. 2011;123(2):109-19.

31. Fuller A, Hetem RS, Meyer LCR, Maloney SK. Angularis oculi vein blood flow modulates the magnitude but not the control of selective brain cooling in sheep. Am J Physiol Regul Integr Comp Physiol. 2011;300(6):R1409-1417.

32. Mitchell J, Thomalla L, Mitchell G. Histological studies of the dorsal nasal, angularis oculi, and facial veins of sheep (Ovis aries). J Morphol. 1998;237(3):275-81.

33. Simpson S, King JL. Localisation of the motor area in the sheep. Exp Physiol. 1911;4(1):53-65.

34. Clarke PG, Whitteridge D. The cortical visual areas of the sheep. J Physiol 1976;256(3):497-508

35. Alpert GF, Handwerker D, Sun FT, D’Esposito M, Knight RT. Spatio-temporal information analysis of event-related BOLD responses. Neurolmage. 2007;34(4):1545-61.

36. Hillman EMC. Coupling mechanism and significance of the BOLD signal: a status report. Annu Rev Neurosci. 2014;37(1):161-81.

37. Wager TD, Vazquez A, Hernandez L, Noll DC. Accounting for nonlinear BOLD effects in $\mathrm{FMRI}$ : parameter estimates and a model for prediction in rapid event-related studies. Neurolmage. 2005;25(1):206-18.

38. Shen $Q$, Ren H, Duong TQ. CBF, BOLD, CBV, and CMRO(2) fMRI signal temporal dynamics at 500-msec resolution. J Magn Reson Imaging. 2008;27(3):599-606.

39. Yang Y, Gu H, Stein EA. Simultaneous MRI acquisition of blood volume, blood flow, and blood oxygenation information during brain activation. Magn Reson Med. 2004;52(6):1407-17.

40. Lahti KM, Ferris CF, Li F, Sotak CH, King JA. Comparison of evoked cortical activity in conscious and propofol-anesthetized rats using functional MRI. Magn Reson Med. 1999;41(2):412-6. 
41. Peeters RR, Tindemans I, De Schutter E, Van der Linden A. Comparing BOLD fMRI signal changes in the awake and anesthetized rat during electrical forepaw stimulation. Magn Reson Imaging. 2001;19(6):821-6.

42. Tsurugizawa $T$, Uematsu $A$, Uneyama $H$, Torii $K$. Effects of isoflurane and alpha-chloralose anesthesia on BOLD fMRI responses to ingested Lglutamate in rats. Neuroscience. 2010;165(1):244-51.

43. Booke M, Armstrong C, Hinder F, Conroy B, Traber LD, Traber DL. The effects of propofol on hemodynamics and renal blood flow in healthy and in septic sheep, and combined with fentanyl in septic sheep. Anesth Analg. 1996;82(4):738-43.

44. Lin H-C, Purohit RC, Powe TA. Anesthesia in sheep with propofol or with xylazine-ketamine followed by halothane. Vet Surg. 1997;26(3):247-52.

45. Myburgh JA, Upton RN, Grant C, Martinez A. Epinephrine, norepinephrine and dopamine infusions decrease propofol concentrations during continuous propofol infusion in an ovine model. Intensive Care Med. 2001;27(1):276-82

46. Brander A, Kataja A, Saastamoinen A, Ryymin P, Huhtala H, Öhman J, et al. Diffusion tensor imaging of the brain in a healthy adult population: Normative values and measurement reproducibility at $3 \mathrm{~T}$ and $1.5 \mathrm{~T}$. Acta Radiol. 2010;51(7):800-7.

47. Yarnykh VL, Yuan C. T1-insensitive flow suppression using quadruple inversion-recovery. Magn Reson Med. 2002;48(5):899-905.

48. Morelli JN, Runge VM, Ai F, Attenberger U, Vu L, Schmeets SH, et al. An image-based approach to understanding the physics of MR artifacts. Radiographics. 2011;31(3):849-66.

49. Matta BF, Heath KJ, Tipping K, Summors AC. Direct cerebral vasodilatory effects of sevoflurane and isoflurane. Anesthesiology. 1999;91(3):677-80.

50. Reiz S, Bålfors E, Sørensen MB, Ariola S, Friedman A, Truedsson H. Isoflurane-a powerful coronary vasodilator in patients with coronary artery disease. Anesthesiology. 1983;59(2):91-7.

51. Triantafyllou C, Hoge RD, Krueger G, Wiggins CJ, Potthast A, Wiggins GC et al. Comparison of physiological noise at 1.5 T, $3 \mathrm{~T}$ and $7 \mathrm{~T}$ and optimization of fMRI acquisition parameters. Neurolmage. 2005;26(1):243-50.

52. Friston KJ, Holmes AP, Worsley KJ. How many subjects constitute a study? Neurolmage. 1999;10(1):1-5.

53. Forman SD, Cohen JD, Fitzgerald M, Eddy WF, Mintun MA, Noll DC. Improved assessment of significant activation in functional magnetic resonance imaging (fMRI): use of a cluster-size threshold. Magn Reson Med. 1995:33(5):636-47.

54. Song X-W, Dong Z-Y, Long X-Y, Li S-F, Zuo X-N, Zhu C-Z, et al. REST: A toolkit for resting-state functional magnetic resonance imaging data processing. PLOS ONE. 2011;6(9):e25031.

55. Alexander AL, Lee JE, Lazar M, Field AS. Diffusion tensor imaging of the brain. Neurotherapeutics. 2007:4(3):316-29.

56. Tournier JD, Calamante F, Connelly A. MRtrix: Diffusion tractography in crossing fiber regions. Int J Imaging Syst Technol. 2012;22(1):53-66.

57. Farquharson S, Tournier JD, Calamante F, Fabinyi G, Schneider-Kolsky M, Jackson GD, et al. White matter fiber tractography: why we need to move beyond DTI. J Neurosurg. 2013;118(6):1367-77.

\section{Submit your next manuscript to BioMed Central and take full advantage of:}

- Convenient online submission

- Thorough peer review

- No space constraints or color figure charges

- Immediate publication on acceptance

- Inclusion in PubMed, CAS, Scopus and Google Scholar

- Research which is freely available for redistribution 